\title{
Growth and Structure of Crystalline Silica Sheet on Ru(0001)
}

\author{
D. Löffler, ${ }^{1}$ J. J. Uhlrich, ${ }^{1}$ M. Baron, ${ }^{1}$ B. Yang, ${ }^{1}$ X. Yu, ${ }^{1}$ L. Lichtenstein, ${ }^{1}$ L. Heinke, ${ }^{1}$ C. Büchner, ${ }^{1}$ M. Heyde, ${ }^{1}$ \\ S. Shaikhutdinov, ${ }^{1, *}$ H.-J. Freund, ${ }^{1}$ R. Włodarczyk ${ }^{2}$ M. Sierka, ${ }^{2, *}$ and J. Sauer ${ }^{2}$ \\ ${ }^{1}$ Department of Chemical Physics, Fritz. Haber Institute of the Max Planck Society, Faradayweg 4-6, 14195 Berlin, Germany \\ ${ }^{2}$ Institute of Chemistry, Humboldt University, Brook-Taylor-Strasse 2, 12489 Berlin, Germany \\ (Received 9 July 2010; revised manuscript received 23 August 2010; published 30 September 2010)
}

Thin $\mathrm{SiO}_{2}$ films were grown on a $\mathrm{Ru}(0001)$ single crystal and studied by photoelectron spectroscopy, infrared spectroscopy and scanning probe microscopy. The experimental results in combination with density functional theory calculations provide compelling evidence for the formation of crystalline, double-layer sheet silica weakly bound to a metal substrate.

PACS numbers: 68.35.- $\mathrm{p}$, 68.47.Gh, 68.55.- a

Silicon dioxide $\left(\mathrm{SiO}_{2}\right)$ plays a key role in many modern technologies and applications that range from insulating layers in integrated circuits to supports for metal and oxide clusters in catalysts. For better understanding of structureproperty relationships on silica-based materials, particularly of reduced dimensions, thin silica films grown on metal single crystal substrates are suggested as suitable model systems that allow the facile application of many "surface science" techniques. It has recently been shown that crystalline silica films and nanowires can be grown on $\mathrm{Mo}(112)$ [1-5]. The ultrathin film consists of a monolayer honeycomblike network of corner-sharing $\left[\mathrm{SiO}_{4}\right]$ tetrahedra, thus resulting in a $\mathrm{SiO}_{2.5}$ stoichiometry of the film. The $\mathrm{Si}$ atoms in these films can be partly substituted by $\mathrm{Al}$ in the course of preparing metal supported aluminosilicate films [6], which is the first step towards experimental modeling of catalytic centers in zeolitelike materials. However, attempts to grow thicker silica films on the Mo substrates resulted in amorphous structures [7-9], most likely due to the formation of strong Si-O-Mo bonds at the interface that govern the growth mode [9]. Recently, the preparation of crystalline silica films on other supports such as $\mathrm{Pd}(100)$ [10] and $\mathrm{Ni}(111)$ [11] has been reported. However, the atomic structure of the films, film surface termination, and the nature of the silica-metal interface were not determined.

In this Letter, we report on the preparation and the atomic structure of well-defined silica films on $\mathrm{Ru}(0001)$. The experimental results, obtained by photoelectron and vibrational spectroscopies and high-resolution scanning probe microscopy, are complemented by density functional theory calculations which together provide compelling evidence for the formation of a double-layer sheet silicate, with a $\mathrm{SiO}_{2}$ stoichiometric composition, weakly bound to a metal support. The results open new perspectives for employing a "surface science" approach to understand the reactivity of silicate surfaces consisting of hydrophobic SiO-Si bonds, such as those of microporous all-silica zeolites [12]. Also, these films can be used as model supports for catalytically active metal and oxide clusters $[4,13]$.
The experiments were performed in an ultrahigh vacuum chamber equipped with low energy electron diffraction (LEED) and Auger electron spectroscopy, x-ray photoelectron spectroscopy (XPS), infrared reflection absorption spectroscopy (IRAS), and scanning tunneling microscopy (STM). Atomically resolved atomic force microscopy (AFM) and STM images were recorded in a second chamber equipped with LEED, Auger electron spectroscopy, and a home-built microscope operating at $4 \mathrm{~K}$ [14]. Additional high-resolution XPS measurements were carried out at the UE52-PGM beamline at the BESSY II (Berlin) synchrotron facility.

The silica films were grown on a $\mathrm{Ru}(0001)$ surface that shares the hexagonal symmetry of the monolayer silica film obtained on $\mathrm{Mo}(112)$. Note, that a structurally similar, hexagonal graphene overlayer readily grows on $\mathrm{Ru}(0001)$ [15]. In addition, the $\mathrm{Ru}(0001)$ surface can form ordered silicide structures $[16,17]$ which could, in principle, lead to a stronger adhesion of silica film to a metal substrate. Finally, the Ru-O bond is much weaker than the Mo-O bond, the formation of which on a Mo(112) substrate as a Si-O-Mo linkage seems to limit the growth of crystalline silica [9].

The preparation includes several steps. The clean $\mathrm{Ru}(0001)$ surface was precovered with oxygen upon exposure to $1 \times 10^{-7}$ mbar $\mathrm{O}_{2}$ at $373 \mathrm{~K}$ for 5 min forming a $(2 \times 1) \mathrm{O}-\mathrm{Ru}(0001)$ surface [18]. Then $\sim 1.3 \mathrm{ML}$ (1 monolayer or $1 \mathrm{ML}=1.4 \times 10^{15}$ atoms $/ \mathrm{cm}^{2}$ ) of Si was deposited at $630 \mathrm{~K}$ at the same pressure of $\mathrm{O}_{2}$. The absence of diffraction spots in LEED suggests that the silica deposits are amorphous and, in addition, not fully oxidized to $\mathrm{Si}^{4+}$ as revealed by XPS. Therefore, the films were further annealed in $2 \times 10^{-6} \mathrm{mbar}_{2}$ at $\sim 1140 \mathrm{~K}$ for $15 \mathrm{~min}$. The high temperature oxidation was necessary to make the film ordered: The resulting films showed a $(2 \times 2)$ LEED pattern shown in Fig. 1(a), where the $(2 \times 2)$ diffraction spots were significantly brighter than those from the $(2 \times 1) \mathrm{O}-\mathrm{Ru}(0001)$ surface.

The XPS spectra, measured both at normal and grazing emissions, revealed only one chemical state of $\mathrm{Si}$, with the 


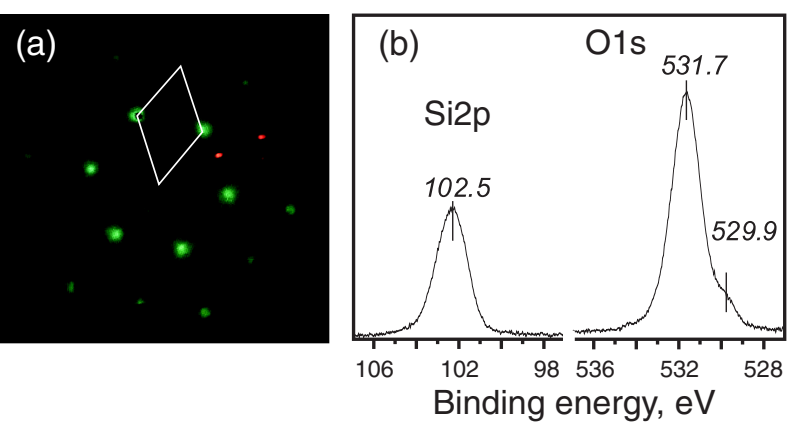

FIG. 1 (color online). (a) LEED pattern of the silica film grown on $\operatorname{Ru}(0001)(E=60 \mathrm{eV})$ where the $(2 \times 2)-\mathrm{Ru}(0001)$ unit cell is indicated. (b) Photoelectron spectra $(h \nu=$ $1253.6 \mathrm{eV}$ ) of the $\mathrm{Si} 2 p$ and $\mathrm{O} 1 s$ core levels.

binding energy (BE) of the Si $2 p$ level of $102.5 \mathrm{eV}$ (referenced to $\mathrm{Au} 4 f_{7 / 2}=84.0 \mathrm{eV}$ ) falling into the region corresponding to $\mathrm{Si}^{4+}$ [19-21], taking into account the screening effects of a metal substrate that effectively lowers the BEs in ultrathin oxide films. Also, the XPS results exclude silicide formation (as it was the case for the preparation of silica films on $\mathrm{Ni}(111)$ [11]). The integral amounts of $\mathrm{Si}$ in the films were determined to be approximately twice that of the monolayer silica film on $\operatorname{Mo}(112)$ used as a reference material with a well-established structure [2,3].

The corresponding $\mathrm{O} 1 \mathrm{~s}$ spectra showed a peak at $531.7 \mathrm{eV}$ and a shoulder at $529.9 \mathrm{eV}$, with the ratio between two signals of $\sim 12: 1$. [For comparison, the topmost ( $\mathrm{Si}-\mathrm{O}-\mathrm{Si})$ and interfacial ( $\mathrm{Si}-\mathrm{O}-\mathrm{Mo})$ oxygen species in $\mathrm{SiO}_{2.5} / \mathrm{Mo}(112)$ films exhibited the BEs at 532.5 and $531.3 \mathrm{eV}$, respectively, with the signal ratio $3: 2[2,3]$. The additional oxygen species having bonds only to the Mo(112) surface in the so-called "O-rich" structures [22] showed a weak signal at $530.6 \mathrm{eV}$, i.e., at the BEs close to that observed on $\mathrm{O} / \mathrm{Mo}(112)$.] The position of a shoulder at $529.9 \mathrm{eV}$ for silica films on $\mathrm{Ru}$ in fact coincides with $\mathrm{O} 1 s$ peak position observed for the $\mathrm{O} / \mathrm{Ru}(0001)$ surface prior to the Si deposition. Its intensity depends on film preparation and is considerably reduced upon annealing in ultrahigh vacuum at $1000 \mathrm{~K}$. In addition, the signal diminishes at grazing emission, thus pointing to the "subsurface" nature of related species. Therefore, we have tentatively assigned the $529.9 \mathrm{eV}$ signal to $\mathrm{O}$ species chemisorbed on $\mathrm{Ru}$ underneath the silica film. These amounts to $\sim \frac{1}{4} \mathrm{ML}$, at most, as calibrated via the $(2 \times 1) \mathrm{O}-\mathrm{Ru}(0001)$ surface.

Immediately after $\mathrm{Si}$ deposition the IRA spectrum is dominated by the signal centered at $\sim 1230 \mathrm{~cm}^{-1}$, which has previously been observed on amorphous silica films grown on metals and assigned to the asymmetric longitudinal-optical phonon mode [8]. Upon high temperature annealing, the band gradually shifts towards higher wave numbers, and a new feature develops at low frequencies, ultimately resulting in the spectrum exhibiting two very sharp peaks, at 1302 and $692 \mathrm{~cm}^{-1}$, as shown in Fig. 2. To the best of our knowledge, the band at

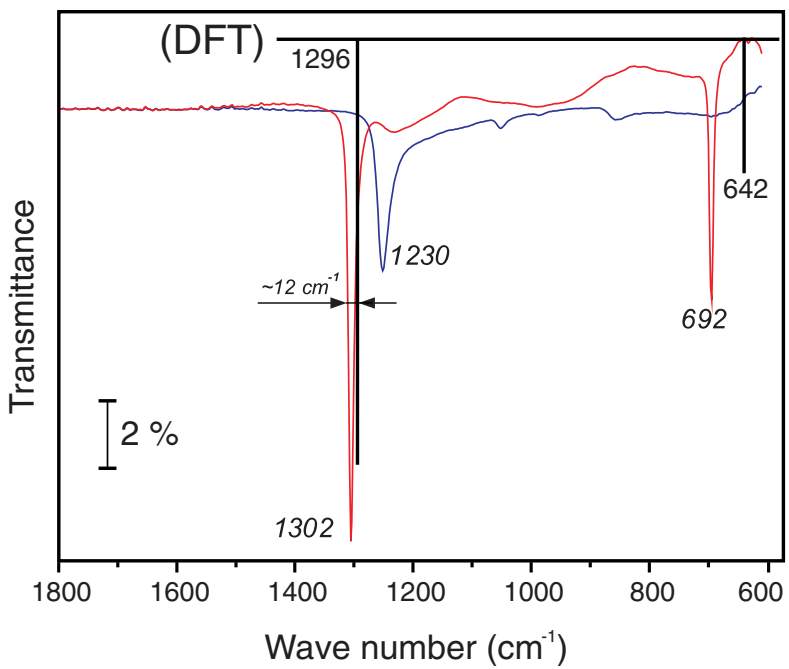

FIG. 2 (color online). IRA spectra of the silica film on $\mathrm{Ru}(0001)$ as deposited at $630 \mathrm{~K}$ [dark grey (blue) curve] and oxidized at $1140 \mathrm{~K}$ [grey (red) curve]. The bars show the position and relative intensity of the IR active vibrations calculated by DFT for the structure shown in Fig. 4(a).

$\sim 1300 \mathrm{~cm}^{-1}$ has never been observed on silica films and crystals $[2,7,8,23-26]$. In addition, the band is by about $250 \mathrm{~cm}^{-1}$ higher than that of Si-O-Mo stretching vibrations on $\mathrm{SiO}_{2} / \mathrm{Mo}(112)$ [2]. Therefore, the band can hardly be assigned to the Si-O-Ru interface. This is also consistent with the XPS results showing a minority of $\mathrm{O} / \mathrm{Ru}$ species. In the ${ }^{18} \mathrm{O}$-labeled films, the bands redshift, i.e., to $1247 \mathrm{~cm}^{-1}\left(\Delta \nu=55 \mathrm{~cm}^{-1}\right)$, and $664 \mathrm{~cm}^{-1}(\Delta \nu=$ $28 \mathrm{~cm}^{-1}$ ), respectively, in perfect agreement with the values predicted on the basis of the reduced masses of a $\mathrm{Si}-\mathrm{O}-\mathrm{Si}$ oscillator. Since only vibrations with the dynamic dipole moment normal to the metal surface contribute to an IRAS signal [27], the very high intensity of the $1302 \mathrm{~cm}^{-1}$ band (almost 10\%) suggests this band to be associated with the Si-O-Si bonds oriented perpendicular to the surface.

Furthermore, we have performed high-resolution STM and AFM studies. Both techniques showed wide terraces, separated by steps of $\sim 2 \AA$ in height, which correspond to the monoatomic steps of $\mathrm{Ru}(0001)$ [Fig. 3(a)]. Atomically resolved images revealed the hexagonal structure with a $5.5 \AA$ periodicity. In Fig. 3(a), the hexagons are formed by six atomic protrusions with an additional contribution in the center. However, the image contrast is bias- as well as tip-dependent, as shown in Fig. 3(c), where the protrusions in the STM data indicate a different symmetry, revealing a chemical sensitivity in the observed imaging contrast. Note that STM images similar to that shown in Fig. 3(c), but not in Fig. 3(b), can be observed for the $3 \mathrm{O}(2 \times 2)-\mathrm{Ru}(0001)$ surface reported in [28]. This finding suggests a similar oxygen arrangement of the topmost layer of silica. In the frequency modulated AFM image, shown in Fig. 3(d), the hexagons are formed by six depressions, and the image exhibits apparently the inverse contrast to the STM image shown in Fig. 3(c). 

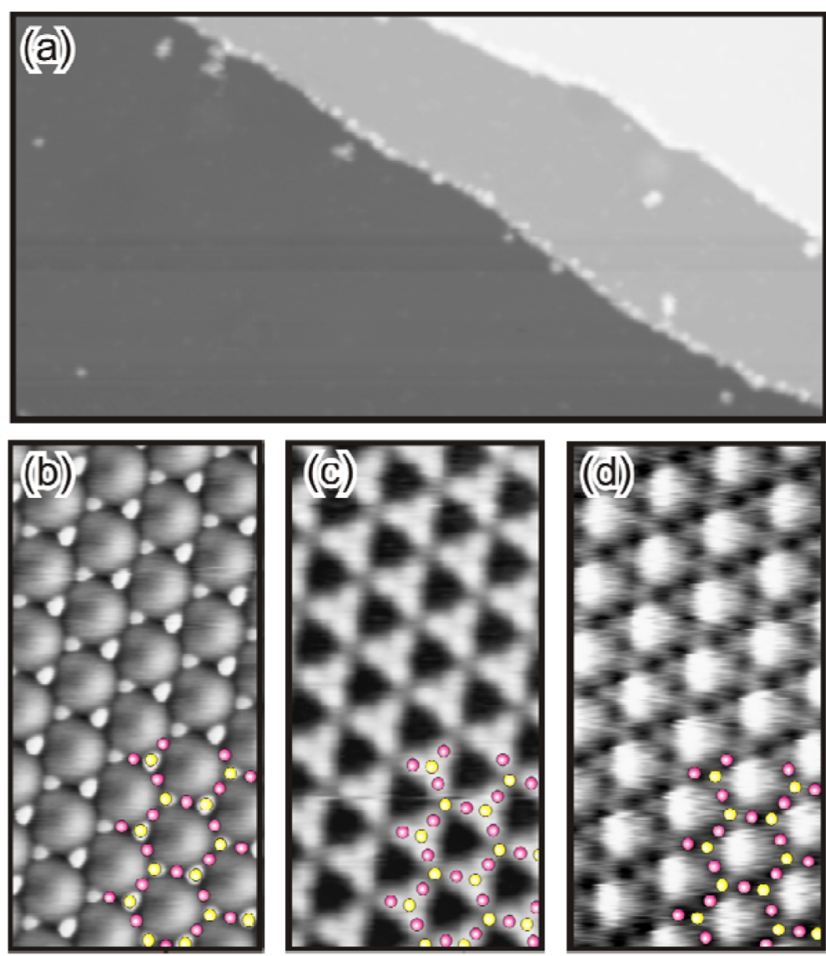

o silicon

- oxygen

FIG. 3 (color online). (a)-(c) STM images of silica film grown on $\mathrm{Ru}(0001)$. Image size: $100 \mathrm{~nm} \times 50 \mathrm{~nm} \quad$ (a) and $2 \mathrm{~nm} \times 4 \mathrm{~nm}$ (b),(c); the bias voltage and current are $0.1 \mathrm{~V}$ and $0.1 \mathrm{nA}$, respectively. (d) FM-AFM image, $2 \mathrm{~nm} \times 4 \mathrm{~nm}$. The frequency shift is $-3 \mathrm{~Hz}$, and the bias voltage is $1.5 \mathrm{~V}$. The images (b)-(d) are partially superimposed with the top view of the models shown in Fig. 4.

According to the $(2 \times 2)$ LEED pattern, the surface lattice constant of the silica film on $\mathrm{Ru}(0001)$ is $5.42 \AA(=2 \times 2.71 \AA)$, which coincides with the periodicity observed by STM and AFM. This value is very close to that observed for the honeycomblike structure of monolayer silica film on $\operatorname{Mo}(112)$, i.e., 5.2-5.5 $\AA$ [2]. Therefore, the same structural motif could be applied to the $\mathrm{Ru}(0001)$ support. Taking into account that silica films formed on Ru possess approximately twice as much $\mathrm{Si}$ as on $\mathrm{Mo}(112)$, we propose that the film is composed of two layers of cornersharing $\left[\mathrm{SiO}_{4}\right]$ tetrahedra bonded together by a linking oxygen which acts as a plane of mirror symmetry [see Fig. 4]. Such a hexagonal prism slab, which is similar to

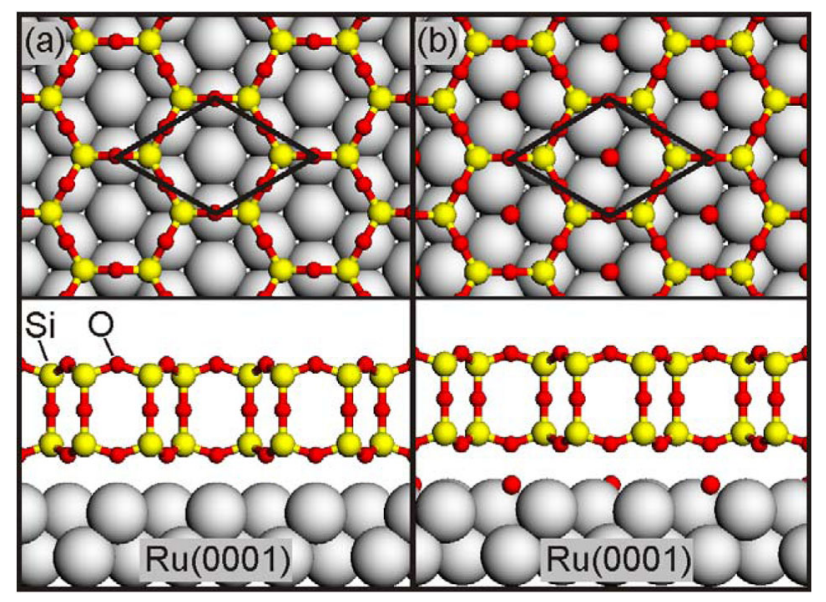

FIG. 4 (color online). Top and side views of the (a) "O-poor" and (b) "O-rich" silica films on $\mathrm{Ru}(0001)$. The $(2 \times 2)$ unit cell is indicated.

double layer structures found in sheet silicates [12], has been proposed as a model for hydrophobic silica surfaces [29]. It was previously considered as a possible model for the silica film on $\operatorname{Mo}(112)$, but discarded because of disagreement with the experimental results [3]. This structure possesses a Si-O-Si linkage normal to the surface suggested by the IRAS results. The silica sheet, having no dangling bonds on either side, would weakly interact with $\mathrm{Ru}$, in agreement with the XPS results showing only one chemical state of $\mathrm{Si}$, the absence of surface silicides, and a minority of of $\mathrm{O}$ species having bonds to $\mathrm{Ru}$. In analogy with the $\mathrm{SiO}_{2.5} / \mathrm{Mo}(112)$ films, the presence of $\mathrm{O} / \mathrm{Ru}$ species can be interpreted in terms of "O-rich" structures to discriminate from the "O-poor" structure where these species are missing.

The models were tested by density functional theory (DFT) including semiempirical dispersion correction $(\mathrm{DFT}+\mathrm{D})$ (see supplementary material [30]). First, we have considered an "O-poor" film where the double silica sheet is placed over the clean $\mathrm{Ru}(0001)$ surface. The only one stable structure found is shown in Fig. 4(a). In this model, all $\mathrm{O}$ atoms in the lowest layer are located above the surface $\mathrm{Ru}$ atoms. The calculated adhesion energy of the silica sheet to the $\mathrm{Ru}(0001)$ support is $3.1 \mathrm{~kJ} \mathrm{~mol}^{-1} \AA^{-2}$, with the main contribution coming from the dispersion term. Table I shows that the interaction energy between

TABLE I. DFT + D adhesion energy $\left(\mathrm{kJ} \mathrm{mol}^{-1} \AA^{-2}\right)$ of the "O-poor" and "O-rich" silica films on $\mathrm{Ru}(0001)$ [shown in Fig. 4] compared to interlayer interaction energies in $\mathrm{V}_{2} \mathrm{O}_{5}$ and graphite.

\begin{tabular}{llccc}
\hline \hline & $\mathrm{SiO}_{2} / \mathrm{Ru}(0001)$ & $\mathrm{SiO}_{2} /(2 \times 2) \mathrm{O}-\mathrm{Ru}(0001)$ & $\mathrm{V}_{2} \mathrm{O}_{5}{ }^{\mathrm{a}}$ & Graphite $^{\mathrm{a}}$ \\
\hline $\mathrm{DFT}+\mathrm{D}$ & -3.09 & -2.38 & -1.89 & -3.14 \\
$\mathrm{DFT}^{\mathrm{b}}$ & 0.75 & 0.98 & -0.35 & 1.4 \\
$\mathrm{D}^{\mathrm{c}}$ & -3.84 & -3.36 & -1.54 & -4.54 \\
\hline \hline
\end{tabular}

${ }^{\mathrm{a}}$ Calculated using the input data of Ref. [31].

${ }^{b}$ DFT contribution using the structure obtained with DFT + D.

${ }^{\mathrm{c}}$ Dispersion contribution. 
the silica layer and the metal surface is comparable to that of graphite and considerably higher than between layers in $\mathrm{V}_{2} \mathrm{O}_{5}$.

The phonon spectrum, calculated for this structure, revealed only two IR active modes above $600 \mathrm{~cm}^{-1}$ measured in the experiments [see Fig. 2]. The most intense mode at $1296 \mathrm{~cm}^{-1}$ is an in-phase combination of asymmetric Si-O-Si stretching vibrations of the Si-O-Si linkage between two layers. The second mode at $642 \mathrm{~cm}^{-1}$ is a combination of symmetric Si-O-Si stretching vibrations of $\mathrm{Si}-\mathrm{O}-\mathrm{Si}$ bonds nearly parallel to the surface. Figure 2 demonstrates that not only the positions but also relative intensities of the vibrations are in very good agreement with the experimental results.

Furthermore, the calculated BEs of the O $1 s$ core levels for the different $\mathrm{O}$ species in this structure showed nearly equal values with the largest difference of $\sim 0.2 \mathrm{eV}$. This finding is another indication that the weak, $529.9 \mathrm{eV}$ signal observed in the XP spectra [Fig. 1] is due to oxygen in direct interaction with Ru.

Various models of the "O-rich" film were created by placing the double silica sheet in different positions over the $(2 \times 2) \mathrm{O}-\mathrm{Ru}(0001)$ surface $\left(\frac{1}{4} \mathrm{ML}\right.$ oxygen coverage). The most stable structure is shown in Fig. 4(b), where the $\mathrm{O}$ atoms in the lowest layer are now located above the hcp hollow sites of $\mathrm{Ru}(0001)$. The adhesion energy for this structure is lower by $0.7 \mathrm{~kJ} \mathrm{~mol}^{-1} \AA^{-2}$ as compared to the film on the clean $\mathrm{Ru}(0001)$ surface (see Table I).

The proposed silica model fits well the hexagonal structure observed by STM and AFM. By superimposing the images with the model, as shown in Figs. 3(b)-3(d), one can explain the atomic contrast, which would, however, depend on the precise tip configuration during the experiment. In Fig. 3(b), the protrusions can be readily assigned to the $\mathrm{Si}$ atoms, whereas they correspond to the topmost $\mathrm{O}$ atoms in Fig. 3(c).

In summary, we have shown that well-defined crystalline $\mathrm{SiO}_{2}$ films, consisting of a double-layer silica sheet, can be grown on a metal support. These films can be used as suitable models for experimental surface science studies of catalytically active species on hydrophobic silica surfaces. The preparation can further be developed for the thin film growth of a wide class of silicate-based catalysts such as aluminum and titanium silicates.

The work was supported by Deutsche Forschungsgemeinschaft (SFB 546). We thank H. Kuhlenbeck, E. Primorac, O. Karslioglu, S. Pohl, and M. Naschitzki for technical assistance at BESSY II.

*Corresponding authors: shaikhutdinov@fhi-berlin .mpg.de; marek.sierka@chemie.hu-berlin.de.

[1] T. Schroeder, M. Adelt, B. Richter, M. Naschitzki, M. Bäumer, and H.-J. Freund, Surf. Rev. Lett. 7, 7 (2000).
[2] J. Weissenrieder, S. Kaya, J.-L. Lu, H.-J. Gao, S. Shaikhutdinov, H.-J. Freund, M.M. Sierka, T.K. Todorova, and J. Sauer, Phys. Rev. Lett. 95, 076103 (2005).

[3] S. Kaya, M. Baron, D. Stacchiola, J. Weissenrieder, S. Shaikhutdinov, T. K. Todorova, M. Sierka, J. Sauer, and H.-J. Freund, Surf. Sci. 601, 4849 (2007).

[4] U. Martinez, L. Giordano, and G. Pacchioni, J. Phys. Chem. B 110, 17015 (2006).

[5] J. Seifert, D. Blauth, and H. Winter, Phys. Rev. Lett. 103, 017601 (2009).

[6] D. Stacchiola, S. Kaya, J. Weissenrieder, H. Kuhlenbeck, S. Shaikhutdinov, H. J. Freund, M. Sierka, T. K. Todorova, and J. Sauer, Angew. Chem., Int. Ed. Engl. 45, 7636 (2006).

[7] J. W. He, X. Xu, J. S. Corneille, and D. W. Goodman, Surf. Sci. 279, 119 (1992).

[8] X. Xu and D. W. Goodman, Appl. Phys. Lett. 61, 774 (1992).

[9] D. J. Stacchiola, M. Baron, S. Kaya, J. Weissenrieder, S. Shaikhutdinov, and H.-J. Freund, Appl. Phys. Lett. 92, 011911 (2008).

[10] Z. Zhang, Z. Jinag, Y. Yao, D. Tan, Q. Fu, and X. Bao, Thin Solid Films 516, 3741 (2008).

[11] M. Kundu and Y. Murata, Appl. Phys. Lett. 80, 1921 (2002).

[12] F. Liebau, Structural Chemistry of Silicates (SpringerVerlag, Berlin, 1985).

[13] J. Döbler, M. Pritzsche, and J. Sauer, J. Phys. Chem. C 113, 12454 (2009).

[14] M. Heyde, G. H. Simon, H. P. Rust, and H.-J. Freund, Appl. Phys. Lett. 89, 263107 (2006).

[15] P. W. Sutter, J.-I. Flege, and E. A. Sutter, Nature Mater. 7, 406 (2008).

[16] Z.H. Lu, T. K. Sham, P. R. Norton, and K. H. Tan, Appl. Phys. Lett. 58, 161 (1991).

[17] M. A. Chesters and A. B. Horn, J. Phys. Condens. Matter 3, S251 (1991).

[18] H. Pfnür, G. Held, and M. Lindroos et al., Surf. Sci. 220, 43 (1989).

[19] F. J. Grunthaner, P. J. Grunthaner, and R. P. Vasquez et al., J. Vac. Sci. Technol. 16, 1443 (1979).

[20] W. Braun and H. Kuhlenbeck, Surf. Sci. 180, 279 (1987).

[21] F. Rochet, C. Poncey, and G. Dufour et al., J. Non-Cryst. Solids 216, 148 (1997).

[22] M. Sierka, T. K. Todorova, and S. Kaya et al., Chem. Phys. Lett. 424, 115 (2006).

[23] J. E. Olsen and F. Shimura, Appl. Phys. Lett. 53, 1934 (1988).

[24] R. M. Almeida and C. G. Pantano, J. Appl. Phys. 68, 4225 (1990).

[25] K. T. Queeney, M. K. Weldon, J. P. Chang, Y. J. Chabal, A. B. Gurevich, J. Sapjeta, and R. L. Opila, J. Appl. Phys. 87, 1322 (2000).

[26] C. T. Kirk, Phys. Rev. B 38, 1255 (1988).

[27] F. M. Hoffmann, Surf. Sci. Rep. 3, 107 (1983).

[28] K. L. Kostov, M. Gsell, P. Jacob, T. Moritz, W. Widdra, and D. Menzel, Surf. Sci. 394, L138 (1997).

[29] P. Ugliengo, B. Civalleri, and S. Tosoni, J. Phys. Chem. C (to be published).

[30] See supplementary material at http://link.aps.org/ supplemental/10.1103/PhysRevLett.105.146104 for the computational details.

[31] T. Kerber, M. Sierka, and J. Sauer, J. Comput. Chem. 29, 2088 (2008). 